Case
Reports

Koray Ak, MD

Selim Isbir, MD

Alper Kepez, MD

Kemal Turkoz, MD

Emre Elci, MD

Sinan Arsan, MD

Key words: Adipose tissue/ pathology; heart septum/ pathology/surgery; hypertrophy/complications; lipomatosis/complications/diagnosis/ surgery; ventricular septum/ pathology

From: Departments of Cardiovascular Surgery (Drs. Ak, Arsan, Elci, and Isbir), Cardiology (Dr. Kepez), and Pathology (Dr. Turkoz),

Marmara University

School of Medicine

34899 Istanbul, Turkey

Address for reprints: Koray Ak, MD, Pendik Egitim ve Arastirma Hastanesi, Marmara Universitesi, Kalp ve Damar Cerrahisi Klinigi 4.kat, Fevzi Cakmak Mah. Ustkaynarca Pendik, 34899 Istanbul, Turkey

E-mail:

korayakmd@gmail.com

(C) 2014 by the Texas Heart ${ }^{\circledR}$ Institute, Houston

\section{Large Lipomatous Hypertrophy of the Interventricular Septum}

We present the case of a 58-year-old woman who had large lipomatous hypertrophy of the interventricular septum, a condition that is reported very infrequently. Preoperative cardiac magnetic resonance images revealed an inhomogeneous, infiltrating mass that was suppressed in fat-suppression mode. The extensive mass was causing right ventricular dysfunction, so we excised it through a right ventricular approach. The findings on histologic analysis of the mass were consistent with lipomatous hypertrophy. The patient died of septic shock on the 28th postoperative day. In addition to the patient's case, we discuss the characteristics and diagnosis of this rare entity. (Tex Heart Inst $\boldsymbol{J}$ 2014;41(2):231-3)

$\mathbf{P}$ rimary lipoid involvement of cardiac structures is reportedly rare, and cardiac lipomas constitute almost all such lipoids. ${ }^{1}$ Cardiac lipomatous hypertrophy is nearly always confined to the interatrial septum (IAS). Its presence in the interventricular septum (IVS), the left ventricle, and the right ventricle (RV) has been detected incidentally in a few patients. ${ }^{2-4}$ We report the case of a patient in whom large cardiac lipomatous hypertrophy was found in the right side of the IVS. In addition, we discuss the nature and diagnosis of this rare entity.

\section{Case Report}

In May 2012, a 58-year-old woman presented for investigation of her 2-year history of progressively worsening dyspnea on exertion. She was admitted to the hospital when a large mass was detected in her IVS. Her medical history was significant for type 2 diabetes mellitus and chronic obstructive pulmonary disease. On admission, she had a New York Heart Association class III functional capacity. Physical examination, an electrocardiogram, and chest radiographs yielded normal results. Auscultation revealed bilateral coarse respiratory sounds. Transthoracic and transesophageal echocardiograms revealed a $3.8 \times 4.2-\mathrm{cm}$ hyperechogenic mass in the IVS, protruding into the RV cavity and the RV outflow tract. Tricuspid valve (TV) insufficiency was moderate. The patient's left ventricular ejection fraction and pulmonary artery pressure were normal. Cardiac magnetic resonance (CMR) images revealed an inhomogeneous mass with high signal intensity on T1 weighting (Figs. 1A and 1B). The mass was suppressed in fat-suppression mode (Fig. 1C). Its borders were not sharply delineated, and an infiltrating pattern between the lipoid mass and the muscular septum was prominent (Fig. 1D). The mass had a broad-based attachment and occupied most of the outflow portion of the RV. Coronary angiograms showed normal coronary arteries. Our working diagnosis was either a benign lipoid mass or an infiltrating liposarcoma of the IVS. Resection of the tumor was scheduled.

After median sternotomy and aortobicaval cannulation, cardiopulmonary bypass (CPB) was started. The patient was cooled to a temperature of $30^{\circ} \mathrm{C}$. After the aorta was cross-clamped and warm-blood cardioplegic solution was administered antegrade, cardiac arrest was established. We explored the mass through a right atriotomy. The mass was extensive, so we performed a vertical right ventriculotomy to facilitate its exposure. The ventricular septum gave off a yellowish reflection and seemed to be irregularly lobulated. We opened the lobulated septal surface and almost completely excised the tumor tissue, without damaging the chordal structures of the TV that arose from the septal surface. After carefully trimming the mass from the septum, we saw only minimal residual tumorous tissue in the regions of infiltration. Intraoperatively, we did not harvest any tumor tissue to later rule out malignancy on frozen 

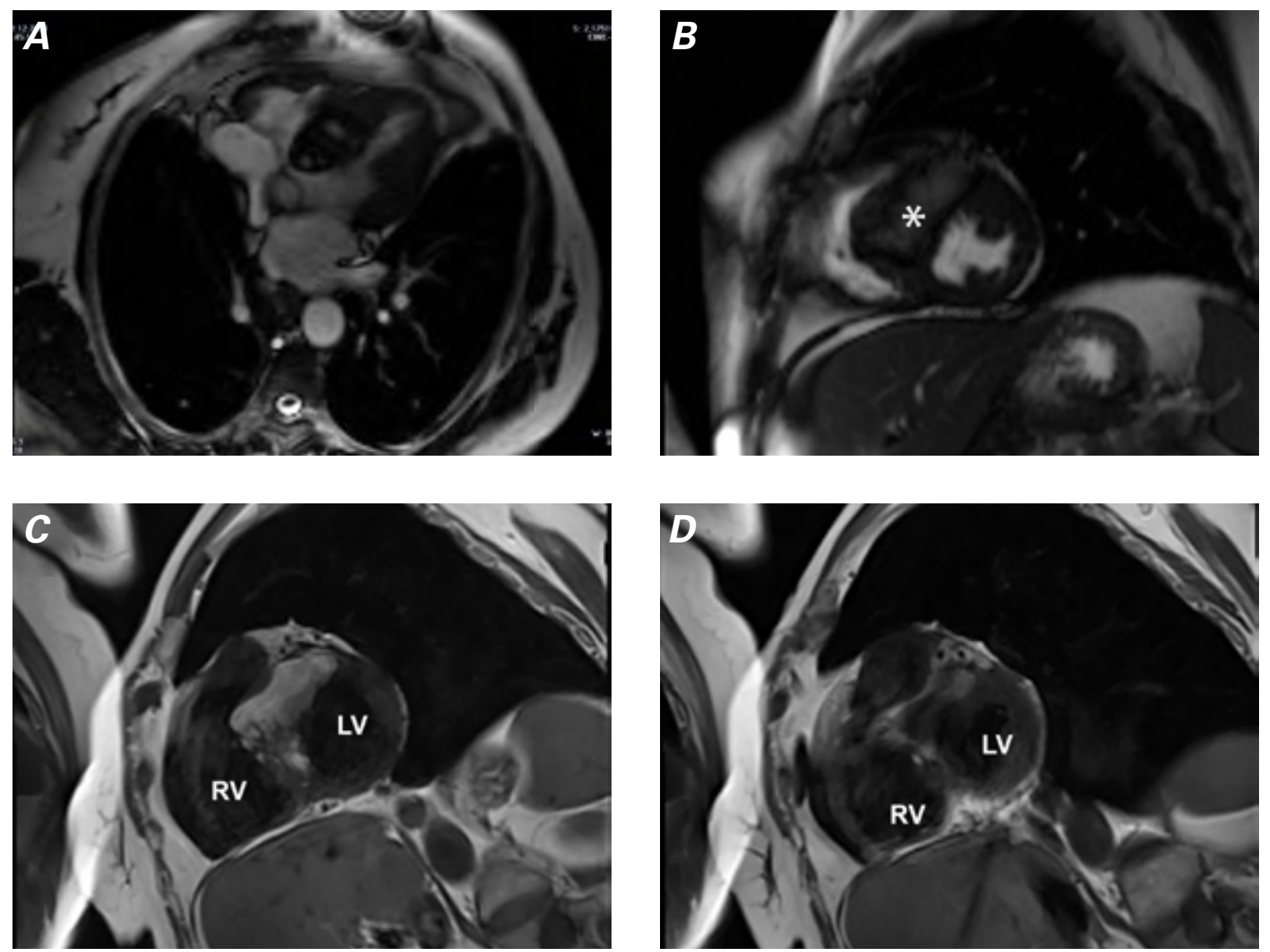

Fig. 1 Cardiac magnetic resonance T1-weighted images in A) 4-chamber view with contrast and B) short-axis view show an inhomogeneous mass (asterisk), with high signal intensity, in the interventricular septum. C) The mass is suppressed in fat-suppression mode. D) In short-axis view, the mass exhibits an irregular, infiltrative pattern.

$L V=$ left ventricle; $R V=$ right ventricle

section. The patient was weaned from CPB uneventfully, and the procedure was completed in a standard fashion. The patient's early postoperative course was complicated by respiratory dysfunction, and she could not be weaned from mechanical ventilation. She died of septic shock on the 28th postoperative day. Histopathologic examination revealed an adipocytic proliferation between the hypertrophic muscle cells of the IVS. No fibrous capsule covering the proliferation was identified in multiple sections (Fig. 2). These findings were consistent with lipomatous hypertrophy of the IVS.

\section{Discussion}

Cardiac lipomatous hypertrophy in the IAS was first described as a distinct entity by Prior in $1964 .^{5}$ Lipomatous hypertrophy of the IAS manifests itself as a nonencapsulated mass of adipose tissue, usually in continuity with the epicardial fat that infiltrates the IAS anterior or superior to the fossa ovalis. Because of the entity's

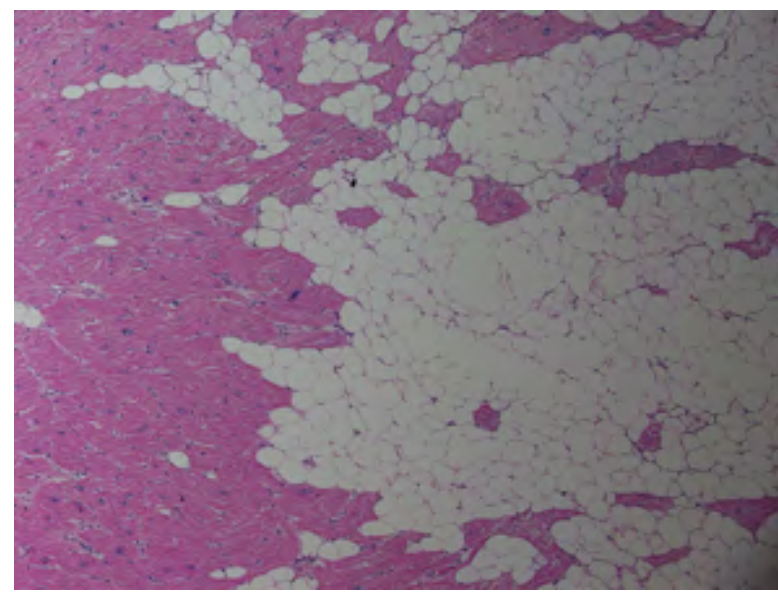

Fig. 2 Photomicrograph shows infiltrating adipose tissue in the interventricular septum. Benign adipocyte proliferation is prominent between hypertrophic muscle cells, and no fibrous encapsulation is present (H\&E, orig. $\times 100)$.

asymptomatic nature, diagnosis is usually made postmortem. Rarely, the lesion is associated with arrhyth- 
mias, recurrent pericardial effusions, heart failure, or sudden death. $3.5,6$

Histologically, cardiac lipomatous hypertrophy differs from a lipoma by its location in the IAS, the absence of a capsule, and the presence of fetal fat. ${ }^{2}$ Its presence in heart tissue outside the IAS has been reported very infrequently. Verberkmoes and colleagues ${ }^{2}$ reported the autopsy finding of extensive lipomatous hypertrophy involving the venae cavae, the atria, and the RV; the surgical procedure had been for a ventricular septal defect and a left atrial myxoma. Nezafati and colleagues ${ }^{3}$ reported the accidental diagnosis on CMR of lipomatous hypertrophy in an asymptomatic patient's IVS and both ventricles. Stephant and associates ${ }^{4}$ reported the case of an asymptomatic patient who had lipomatous hypertrophy in the IVS. In the medical literature, almost all patients with lipomatous hypertrophy in the IVS had small intramyocardial masses buried in the septum, and diagnosis was usually incidental. Because the risk of malignancy is low, surgical treatment is not indicated in asymptomatic patients. In our patient, resection was indicated by the large size of the mass, which protruded into the RV cavity and caused RV dysfunction. The patient's moderate TV insufficiency might be explained by the tumor's displacement of the TV chordal structures arising from the IVS.

The initial diagnostic tool for evaluating primary cardiac masses is 2-dimensional transthoracic echocardiography (TTE); however, accurate tissue characterization might not be possible by this means. Advances in CMR, computed tomography, and 3-dimensional TTE have enabled rapid acquisition of images of the heart with high spatial and temporal resolution and excellent myocardial tissue characterization.$^{7.8}$ In our patient's case, the tumor was identified in the CMR fat-suppression mode as fat tissue. Unlike cardiac lipomas, this septal lipoid mass had an infiltrating pattern with no clear separation zone between it and the myocardium; hence, it is not possible to rule out an infiltrating malignant tumor. It can be challenging to distinguish lipomatous hypertrophic masses or lipomas from well-differentiated liposarcomas with the use of CMR findings, so endomyocardial biopsy can be performed in asymptomatic patients who have suspected lipomatous hypertrophy of the IVS.

\section{References}

1. Miralles A, Bracamonte L, Soncul H, Diaz del Castillo R, Akhtar R, Bors V, et al. Cardiac tumors: clinical experience and surgical results in 74 patients. Ann Thorac Surg 1991;52 (4):886-95.

2. Verberkmoes NJ, Kats S, Tan-Go I, Schonberger JP. Resection of a lipomatous hypertrophic interatrial septum involving the right ventricle. Interact Cardiovasc Thorac Surg 2007;6 (5):654-7.

3. Nezafati MH, Javan H, Maleki MH, Hamedanchi A. Lipomatosis of interventricular septum and both ventricles: an extremely rare pattern. Interact Cardiovasc Thorac Surg 2011; 12(3):511-2.

4. Stephant E, Barthelet M, Leroux PY, Revel D. Images in cardiovascular medicine. Lipomatous hypertrophy of the interventricular septum: echocardiography, cardiac magnetic resonance, and multidetector computerized tomography imaging. Circulation 2008;118(4):e71-2.

5. Prior JT. Lipomatous hypertrophy of cardiac interatrial septum. A lesion resembling hibernoma, lipoblastomatosis and infiltrating lipoma. Arch Pathol 1964;78:11-5.

6. O'Connor S, Recavarren R, Nichols LC, Parwani AV. Lipomatous hypertrophy of the interatrial septum: an overview. Arch Pathol Lab Med 2006;130(3):397-9.

7. O'Donnell DH, Abbara S, Chaithiraphan V, Yared K, Killeen RP, Cury RC, Dodd JD. Cardiac tumors: optimal cardiac MR sequences and spectrum of imaging appearances. AJR Am J Roentgenol 2009;193(2):377-87.

8. Leja MJ, Shah DJ, Reardon MJ. Primary cardiac tumors. Tex Heart Inst J 2011;38(3):261-2. 